\title{
CALIBRATION AND VALIDATION OF AN OVERDAMPED DISPLACEMENT ADAPTIVE PROCEDURE FOR SEISMIC ASSESSMENT OF INFILLED RC FRAMES
}

\author{
F. Barbagallo ${ }^{1}$, M. Bosco ${ }^{1}$, A. Ghersi ${ }^{1}$, E. M. Marino ${ }^{1}$, P.P. Rossi ${ }^{1}$, \\ ${ }^{1}$ 1Department of Civil Engineering and Architecture, University of Catania, via S. Sofia 64, 95125 \\ Catania, Italy \\ e-mail: \{fbarbaga, mbosco, aghersi, emarino, prossi\}@dica.unict.it
}

\begin{abstract}
The main part of the existing buildings in the Mediterranean area is extremely vulnerable to seismic actions. Since a complete renewal of the building heritage is not possible, the seismic assessment of existing structures has gained importance to identify the structural deficiencies and design the most appropriate retrofit interventions. Nonlinear dynamic analysis is widely recognised as the most accurate tool to predict the seismic behaviour of structures. However, it has high computational cost and is not suitable for professional purposes. To provide a tool that reliably assesses the seismic behaviour of structures, with an acceptable computational burden, nonlinear static methods of analysis have been developed.

This paper proposes a multimodal adaptive procedure named overDamped Displacement Adaptive Procedure (D-DAP) for seismic assessment of $R C$ frames. The D-DAP is ruled by two main parameters: the value of top displacement adopted as step size and the number of modes of vibrations deemed significant for the determination of the load vector. Furthermore, the D-DAP is equipped with an equivalent damping law to consider the increase of the energy dissipation due to the cumulated damage in both structural and non-structural members.

The goal of this paper is to determine the values of the displacement step size and the number of modes of vibration necessary to achieve a suitable accuracy by means of the D-DAP. In addition, a new equivalent damping law is calibrated considering also infilled frames. In fact, nonlinear static analysis provided in literature are not optimised to estimate the nonlinear behaviour of infilled frames, despite the well known influence of infill panels on the structural response. A parametrical investigation that includes frames with and without infills is conducted. A set of $54 R C$ frames have been designed to be representative of existing buildings with various levels of seismic deficiencies and analysed. The adopted research methodology assumed the response obtained by Incremental nonlinear Dynamic Analysis (IDA) as target.
\end{abstract}

Keywords: nonlinear static analysis, infill walls, RC frames, seismic assessment. 


\section{INTRODUCTION}

Recently, increasing efforts have been devoted by the scientific research to develop methods of analysis to predict the seismic behaviour of existing structures. Since real structures cannot keep an elastic behaviour under strong earthquakes, an accurate estimation of the seismic performance of existing structures requires the determination of the inelastic deformation experienced by structural members during earthquakes. To this end, the nonlinear dynamic analysis is considered the most reliable tool. However, due to its complexity and high computational cost, this type of analysis is still considered accessible only for experts in seismic engineering and is not suitable for daily professional use.

From the need of a tool that estimates the inelastic deformations of structures subjected to earthquake with relative low computational burden, the scientific research has developed the nonlinear static methods of analysis. Among the approaches available in scientific literature, the Capacity Spectrum Method (CS), proposed by Freeman [1], and the N2 Method, proposed by Fajfar [2], are the most popular. Although the results obtained by these analyses are generally deemed adequate for low-rise plane frames, common nonlinear static analyses do not take into account the contribution of higher modes of vibration to the seismic response, and do not consider the progressive reduction of the structural stiffness due to the nonlinear behaviour of the structure. To improve the level of accuracy, advanced nonlinear static methods of analysis were developed. Among others, innovative approaches are the DAP proposed by Pinho et al. $[3,4]$, and the Advanced N1 method proposed by Lenza et al. [5, 6]. Despite the innovative character of these two latter methods, they still suffer from some approximations: the DAP still requires the use of an equivalent SDOF system for the evaluation of the displacement demand, whilst the Advanced N1 method neglects the effect of the energy dissipation due to the inelastic behaviour of the structure.

An additional important aspect is that existing structures are generally endowed with infill panels. Usually, infills are considered nonstructural elements, and their contribution to the seismic response is "conservatively" neglected. However, frames with infill panels have a significantly larger stiffness, and their dissipative capacities and collapse mechanisms are deeply influenced by the presence and mechanical properties of infills. Despite this, neither the nonlinear static methods of analysis suggested by codes, nor the advanced nonlinear static methods were conceived to estimate accurately the nonlinear behaviour of infilled frames, which may be "unconservatively" predicted.

To overcome these issues, in a previous paper [7] the authors proposed a multimodal adaptive nonlinear static procedure named overDamped Displacement Adaptive Procedure (DDAP). This method was developed by combining the approach by Antoniou and Pinho [3] and Pinho et al. [4] to update the load vector, with the procedure by Lenza et al. [5,6] to associate the peak ground acceleration to the displacement demand. The D-DAP explicitly considered the increase of the energy dissipation due to the cumulated damage in the structure and, to this end, the equivalent viscous damping ratio was updated at each step of the pushover analysis. The goal of this paper is to calibrate the value of the parameters ruling the method, i.e. number of modes to be enveloped and size of the incremental step. In addition, an equivalent damping law is calibrated here on RC framed structures, with and without infills. Then, the efficiency of the calibrated method in predicting the seismic response of RC frames is investigated. To this end, a set of $54 \mathrm{RC}$ frames has been designed to be representative of existing RC buildings, suffering from various types and levels of seismic deficiency. The set of case studies includes frames endowed with infill panels with negligible, medium and large stiffness and strength. The adopted research methodology assumed the response obtained by Incremental nonlinear Dynamic Analysis (IDA) as target. 


\section{THE OVERDAMPED DISPLACEMENT ADAPTIVE PROCEDURE}

The proposed D-DAP is structured in three main steps: (1) definition, application and scaling of the load pattern, (2) association of the displacement demand to the corresponding PGA, (3) overdamping correction. The three steps are repeated until the structural collapse (or a target displacement) is achieved. Figure 1 shows the flowchart of the procedure.

The D-DAP applies a displacement load pattern, that is updated at every step of the analysis according to the modal shapes of the structure at the current step. For this reason, at the beginning of every step of D-DAP a modal response spectrum analysis is run. The modal response spectrum analysis is carried out assuming an elastic spectrum having a reference peak ground acceleration $a_{g, r e f}$ and $5 \%$ equivalent viscous damping ratio. The contributions of $n$ modes of vibration to the seismic response are considered. The displacement $D_{i}$ of the load pattern (at the $\mathrm{i}$-th storey) is calculated as the sum of the interstorey drifts $\Delta_{k}$ from storey 1 to the i-th storey, following the interstorey displacement-based scaling approach suggested in $[3,4]$ :

$$
D_{i}=\sum_{k=1}^{i} \Delta_{k}
$$

The interstorey drift $\Delta_{k}$ is determined at every storey as the SRSS combination of the considered modal shapes. The displacements obtained by Equation 1 are normalised to keep the maximum displacement equal to 1 . Hence, the normalised modal scaling vector $D^{\prime}$ defines the shape, not the magnitude, of the load (or increment of load) vector.

At each step of the analysis, a fixed increase of the top displacement $\Delta D_{t}$ is imposed. The displacement load pattern obtained from the modal response spectrum analysis is scaled so that the displacement at the top storey corresponds to $\Delta D_{t}$. The final load pattern $D_{i, s}$ applied at the current step $s$ at each i-th storey is given by the summation of the displacement vector cumulated up to the previous step $D_{i, s-1}$ and the increase of the loading vector at the current step $\Delta D_{i, s}$.

In order to associate the displacement demand to the corresponding PGA, the following observation is made: as long as the increase of the load pattern is assumed small enough, it can be reasonably assumed that the structure has a linear response within the step $[5,6]$.

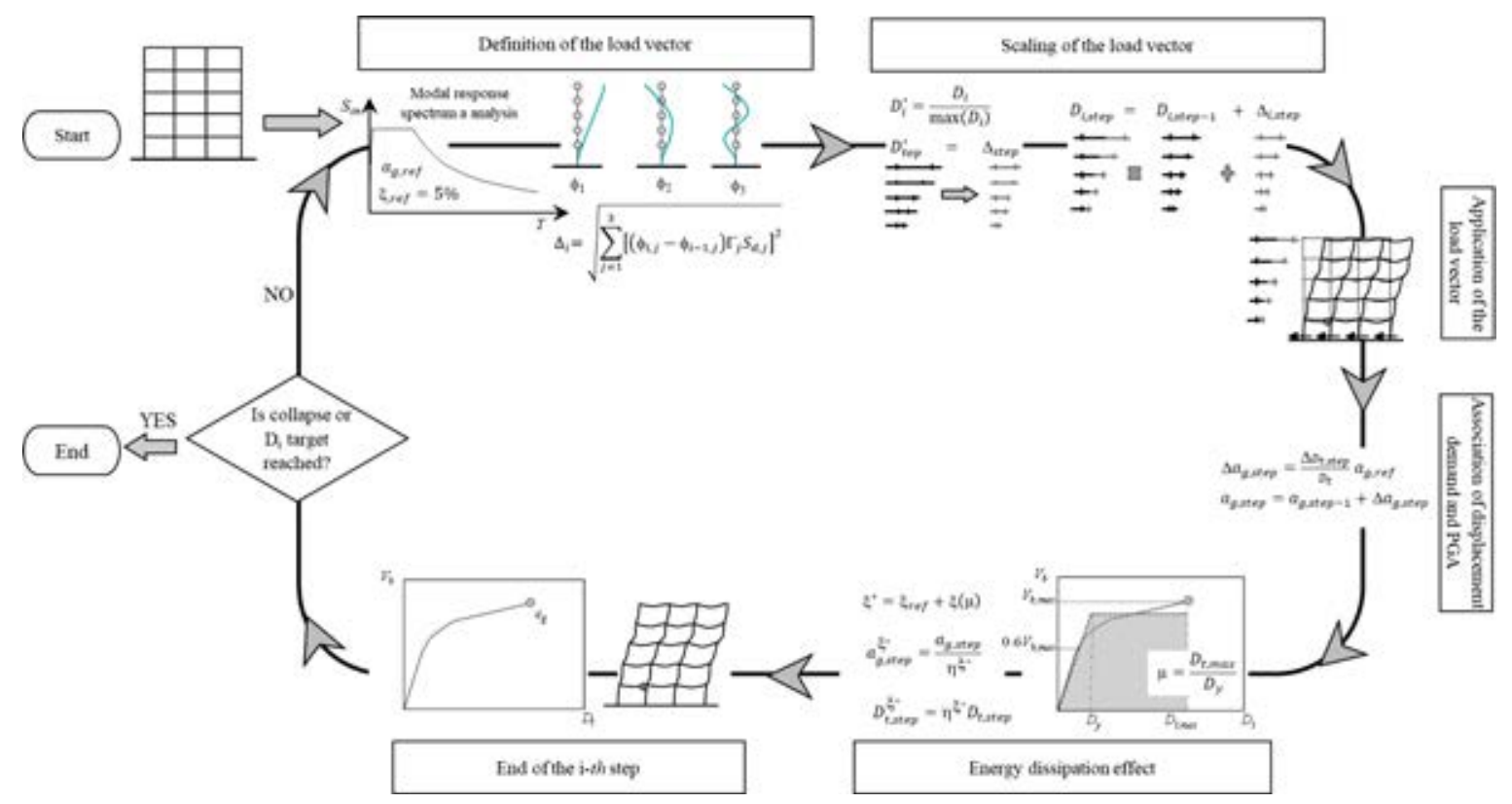

Figure 1 - Flowchart of the D-DAP procedure 
Hence, the increase of PGA $\left(\Delta a_{g, s}\right)$ that has caused the increase of load pattern $\Delta D_{t, s}$ at the step $s$ is evaluated by linear interpolation (note that the increase of load pattern corresponds to the increase of the top displacement of the structure):

$$
\Delta a_{g, s}=\frac{\Delta D_{t, s}}{D_{t}} a_{g, r e f}
$$

where $D_{t}$ is the displacement at the top of the structure provided by the modal response spectrum analysis with response spectrum characterised by the reference acceleration $a_{g, r e f}$. The acceleration $a_{g, s}$ corresponding to the top displacement at the end of step $s$ is:

$$
a_{g, s}=a_{g, s-1}+\Delta a_{g, s}
$$

So far, the increase of acceleration $\Delta a_{g}$, (Eq. 2) has been evaluated with reference to a fix value of the equivalent viscous damping ratio $\xi_{\text {ref. }}$ However, it should be noted that, the equivalent viscous damping ratio increases at every step of the analysis along with the spread of the yielding in the structure. The value of $\xi_{\text {ref }}$ is included in the factor $\eta$, which appears in the equation proposed by EC 8 for the determination of the spectral displacement $S_{d e}$. As $\xi_{\text {ref }}$ is assumed equal to $5 \%$ in both Equations 5 and 6, the energy dissipated by the structure through yielding is not taken into account yet. To account for this, the PGA evaluated by Equation 6 is corrected considering higher values of $\xi_{\text {ref. }}$ Regardless of the fundamental period $T_{1}$ or the type of soil, the ratio of a spectral displacement $S_{d, e}^{5 \%}$ obtained from the response spectra scaled with 5\% damping and the spectral displacement $S_{d, e}^{\xi^{*}}$ obtained from the response spectra scaled with a larger damping ratio $\xi_{\mathrm{eq}}=\xi^{*}$ is equal to the ratio of the corresponding parameters $\eta$ :

$$
\frac{S_{d, e}^{5 \%}}{S_{d, e}^{\xi^{*}}}=\frac{\eta^{5 \%}}{\eta^{\xi^{*}}}
$$

Since $\eta$ is equal to 1 when the damping ratio is $5 \%$ [7] it follows that:

$$
S_{d, e}^{\xi^{*}}=\eta^{\xi *} S_{d, e}^{5 \%}
$$

Since the effect of the equivalent viscous damping is the result of the entire damage cumulated by the structure, from the beginning of the analysis until the considered step, the correction that accounts for the increase of the equivalent damping can be applied straightforwardly to the total PGA at the end of each step of the D-DAP:

$$
a_{g, s}=\frac{a_{g \% t}^{5 \%}}{\eta\left(\xi^{*}\right)}
$$

\section{DESIGN OF CASE STUDIES}

Two sets of RC frames have been created to simulate existing buildings with different features. The first set is composed of frames (named GL) that are drawn from buildings designed to sustain gravity loads only and are representative of typical Italian residential buildings of the seventies. The frames of the second set (named SR) are extracted from RC buildings designed for earthquake resistance in low seismicity areas according to the Italian standards for constructions and seismic resistance [10] in force in the nineties. Two different plan layouts have been considered (Figure 3): in the first plan layout, the unidirectional slab is characterised by reinforcements orientated along the y-direction (Figure 2(a) and (c)), while in the second arrangement the unidirectional slab is parallel to the $\mathrm{x}$-direction (Figure 2 (b) and (d)). All the analysed frames lay along the x-direction. Each set of frames includes three types of frames: frame type 1 and 2 are the central and the outermost frames, respectively, extracted from the buildings where the slab is parallel to y-direction. In consequence of the orientation 
of the deck, frame type 1 supports larger gravity loads than frame type 2 . Frame type 3 is drawn from the building with the slab orientated along the x-direction and supports only the self-weight of beams, columns, infills and the weight of an additional $50 \mathrm{~cm}$ wide stripe of deck. Both the SR frames and the GL frames were designed with five $5.5 \mathrm{~m}$ wide bays and with a number of storeys equal to 3, 6 and 9. The interstorey height is equal to $3.2 \mathrm{~m}$. To investigate the influence of infill panels on the seismic response of buildings, the RC case study frames have been endowed with infill panels, supposing the distribution of the infills uniform along the height of the frame. Particularly, two kinds of infill panels are considered: one with high stiffness and strength, and another having lower stiffness and strength. Furthermore, the case study frames have been considered in the bare configuration as well. These cases represent the limit case of frames having very weak infill panels. In total, $54 \mathrm{RC}$ frames are analysed.

Hereinafter, each frame is identified by a label. The first two letters indicate which set the frame belongs to, the first number indicates the number of storeys and the last number the amount of gravity load. Frames with the largest gravity loads are indicated with 1, frames with the lowest gravity loads are indicated with 3 and the intermediate loaded frames are indicated with 2. The second part of the label is required in the case of infilled frames. Here, the letter I indicates the presence of infill panels and the number indicates the cracking strength of infills in MPa.

The design internal forces of structural members belonging to GL frames are evaluated considering gravity loads only [8]. The internal axial forces $\mathrm{N}$ on columns and the distributed loads on beams are evaluated based on the tributary area concept. The dimensions of the cross sections and the size of steel reinforcements are determined by the allowable stress method [9].

To design the seismic-resistant frames, the regulations for building structures and seismic resistance enforced in Italy in the nineties [10] are followed. The effect of the seismic forces is determined by the lateral force method of analysis supposing that, at the time of construction, the location of the building was classified as low seismicity area.

Details on the design of frames can be found in [7].

\section{CALIBRATION OF PARAMETERS}

In this section, first, an analysis is conducted to calibrate (1) the number of modes to be enveloped and (2) the value of the displacement step size. The goal is to select the values of the optimal ruling parameters that ensure the best accuracy together with the best efficiency of the D-DAP for the case study frames. Hence, once these optimal parameters have been determined, a new equation of the equivalent viscous damping ratio as a function of the period of the first mode of vibration is proposed. The numerical analyses are performed by OpenSees $[11]$.

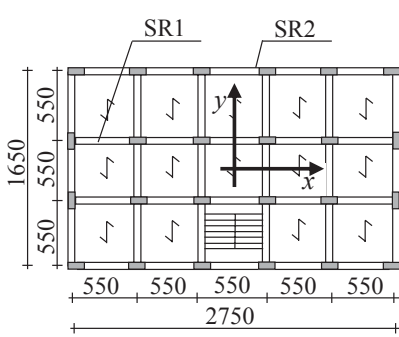

(a)

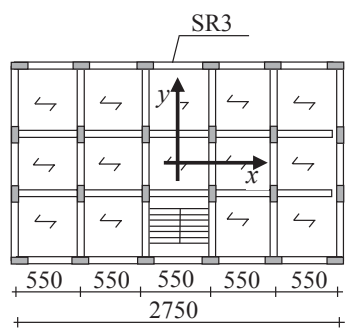

(b)

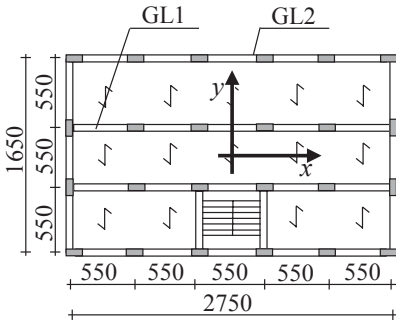

(c)

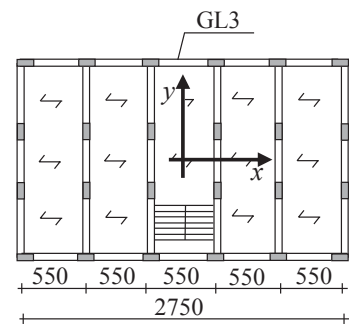

(d)

Figure 2 - Plan layout of buildings (units in $\mathrm{cm}$ ): (a) GL building and (c) SR building with slab perpendicular to longitudinal frames; (b) GL building and (d) SR building with slab parallel to longitudinal frames 


\subsection{Numerical model and seismic input}

The nonlinear response of the case study frames has been investigated by a 2D numerical model with masses concentrated at floor levels. The slab at each level is assumed rigid in its own plane and is simulated by a rigid diaphragm. A leaning column is added to the numerical model to account for $P$ - $\Delta$ effects. For nonlinear dynamic analysis, a Rayleigh viscous damping is used and set at $5 \%$ for the first and the third mode of vibration.

The gravity loads assigned to beams and columns are those specified in EC8 for the seismic design situation. The floor masses are determined as a percentage of the total mass of the deck and are equal to $99.3 \mathrm{t}$ for frames GL1 and GL2, 198.6 for frame GL3 and $106.1 \mathrm{t}$ for frames SR. The gravity load at each level of the leaning column is equal to the seismic weight of the storey of the frame (i.e. the floor mass assigned to frame times 9.81).

Beams and columns of the RC frame are modelled as members constituted by an elastic element with plastic hinges at their ends (Beam With Hinges Element in OpenSees). The length of the plastic hinge is equal to the depth of the cross section and a fibre cross section is assigned to each plastic hinge. The concrete part of the cross section is subdivided into fibres having $5 \mathrm{~mm}$ depth and width equal to the width of the cross section. Single fibres enclosed in the cross section are used to model rebars.

The Mander constitutive law ("Concrete04" uniaxial material) is assigned to concrete fibres [12]. The parameters used for concrete material are summarized in Table 1. Since frames SR are expected to be endowed with dense and well detailed stirrups, the concrete compression strength of the core of the member cross sections is supposed to increase due to the confinement effect of the stirrups. The confinement effect on the core of member cross sections is neglected for frames GL, because RC structures designed without seismic provisions generally have very few stirrups. An elasto-plastic with strain kinematic hardening constitutive law ("Steel01" uniaxial material) is assigned to steel fibres and the values of the mechanical parameters are reported in Table 2.

A "ZeroLength Element" is added at one end of each beam. This element connects the end of the beam to the corresponding node restrained by the rigid deck and is characterized by a low axial stiffness and very large shear and flexural stiffnesses. This expedient allows the beams to deform axially, so as to prevent arising of axial force, which leads fibre modelled

Table 1 Characterization of concrete for nonlinear analysis of frames

\begin{tabular}{|c|c|c|c|c|c|c|c|}
\hline Frame GL & & $f_{c m}(\mathrm{MPa})$ & $\varepsilon_{c 0}$ & $\varepsilon_{c u}$ & $E_{c m}(\mathrm{MPa})$ & $f_{t m}(\mathrm{MPa})$ & $\varepsilon_{t}$ \\
\hline \multirow{2}{*}{3 and 6 storeys } & Core & 28 & 0.002 & 0.0500 & 29961 & 0 & 0 \\
\hline & Cover & 28 & 0.002 & 0.0035 & 29961 & 0 & 0 \\
\hline \multirow{2}{*}{9 storeys } & Core & 33 & 0.002 & 0.0500 & 31477 & 0 & 0 \\
\hline & Cover & 33 & 0.002 & 0.0035 & 31477 & 0 & 0 \\
\hline Frame SR & & $f_{c m}(\mathrm{MPa})$ & $\varepsilon_{c 0}$ & $\varepsilon_{c u}$ & $E_{c m}(\mathrm{MPa})$ & $f_{t m}(\mathrm{MPa})$ & $\varepsilon_{t}$ \\
\hline \multirow{4}{*}{3 and 6 storeys } & Core (columns) & 37 & 0.0054 & 0.0500 & 29961 & 0 & 0 \\
\hline & Cover (columns) & 28 & 0.0020 & 0.0035 & 29961 & 0 & 0 \\
\hline & Core (beams) & 33 & 0.0039 & 0.0500 & 29961 & 0 & 0 \\
\hline & Cover (beams) & 28 & 0.0020 & 0.0035 & 29961 & 0 & 0 \\
\hline \multirow{4}{*}{9 storeys } & Core (columns) & 44 & 0.0055 & 0.0500 & 31477 & 0 & 0 \\
\hline & Cover (columns) & 33 & 0.0020 & 0.0035 & 31477 & 0 & 0 \\
\hline & Core (beams) & 41 & 0.0045 & 0.0500 & 31477 & 0 & 0 \\
\hline & Cover (beams) & 33 & 0.0020 & 0.0035 & 31477 & 0 & 0 \\
\hline
\end{tabular}

Table 2 Characterization of steel for nonlinear analysis of frames

\begin{tabular}{lccc}
\hline & $\boldsymbol{f}_{\text {ym }}(\mathbf{M P a})$ & $\boldsymbol{E}_{\text {sm }}(\mathbf{M P a})$ & $\boldsymbol{b}$ \\
\hline Frame GL & 400 & 210000 & 0.0044 \\
\hline Frame SR & 450 & 210000 & 0.0049 \\
\hline
\end{tabular}


RC beams to an artificial stiffening and strengthening [13]. In the meantime, it ensures the transfer of shear force and bending moment from the beam to the frame node.

Infill panels are modelled as a pair of diagonal trusses without tension resistance. The force-displacement relationship of the diagonal truss is calibrated to replicate the shear forcedrift relationship proposed by Panagiotakos and Fardis [14] and Celarec et al. [15]. This relationship consists of four branches and the stiffness and the value of the maximum force of each branch are determined according to the equations proposed in [15]. Two types of infill panels have been considered here and both of them were supposed to be realized by ceramic bricks, with thickness equal to $20 \mathrm{~cm}$, Young modulus and shear modulus equal to 4130 and $1240 \mathrm{MPa}$, respectively. The so called "strong" infills were characterized by stiffness and cracking strength $(0.28 \mathrm{MPa})$ that were twice the stiffness and strength $(0.14 \mathrm{MPa})$ of the "weak" type of infills. The mechanical properties were taken from the data provided in [15] according to the experimental tests conducted at the University of Pavia [16]. Further details on the adopted infill modelling may be found in [7].

\subsection{Calibration of the number of modes of vibration}

The load vector applied in the D-DAP is evaluated from the envelope of the modal drifts at each storey. In this regard, it is well recognized that the higher the mode of vibration, the least its contribution to the total seismic response. In order to select the number of modes actually significant for the analysed frames, the seismic response of the case study frames has been evaluated by the D-DAP including an increasing number of modes. For each frame, the number of modes of vibration ranged from 1 to the total number of participating modes, equal to the number of storeys. The final step of each analysis has been set at a target top displacement equal to the $5 \%$ of the total height of the frame.

For the sake of simplicity, Figure 3 shows the results of the analysis conducted on frame type GL-1 and SR-1, with 3 and 9 storeys, with and without infills. The seismic responses of

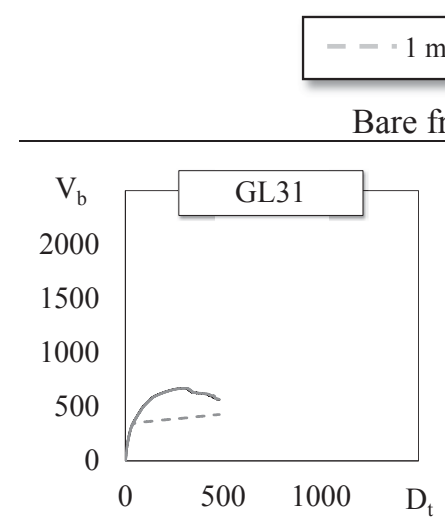

(a)

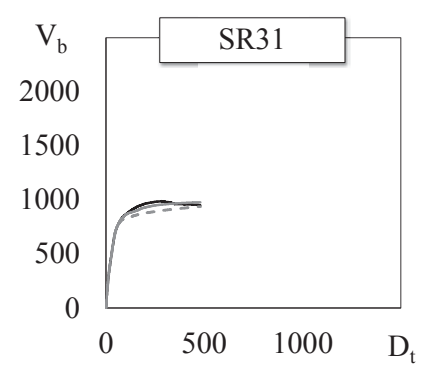

(e)

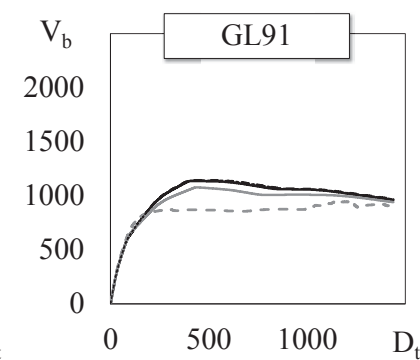

(b)

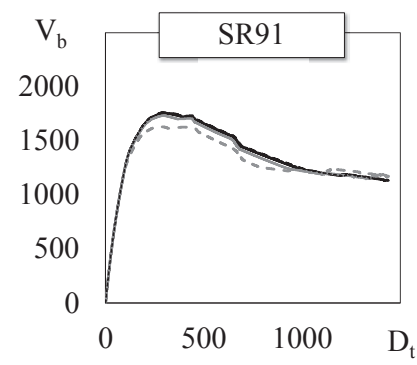

(f)

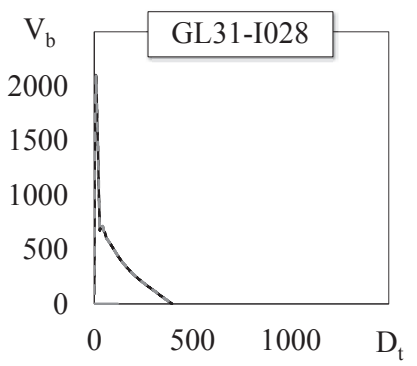

(c)

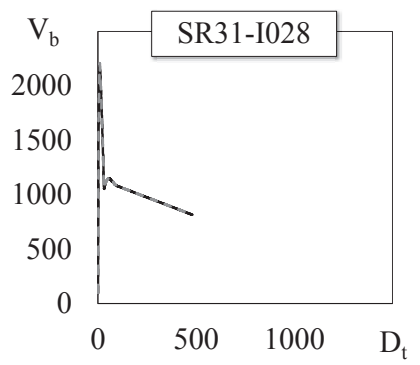

(g)

9 modes

Infilled frames

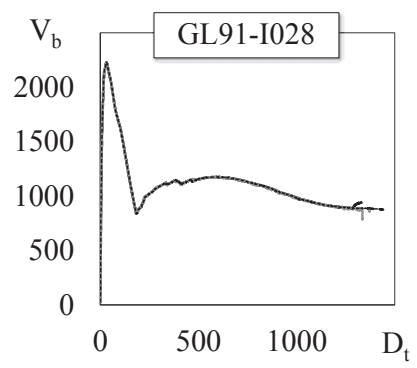

(d)

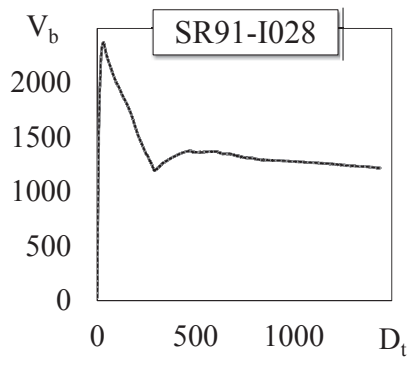

(h)

Figure 3 -Analysis of significant modes of vibration for frame (a) GL31, (b) GL91, (c) GL31-I028, (d) GL91-I028 (e) SR31, (f) SR91, (g) SR31-I028, (h) SR91-I028 
the considered bare frames (Figures (a), (b), (e) and (f)) show that for a fixed value of the top displacement the influence of the higher modes of vibration leads to a larger base shear. In particular, this tendency becomes more significant when the number of storeys increases. Indeed, when the structure yields, the first mode of vibration becomes very flexible and its period increases. As a consequence, the contribution of the first mode to displacements becomes progressively more significant, while its contribution to the internal forces reduces and tends to be negligible. On the contrary, higher modes of vibration are less influenced by yielding of the structure. Thus, although the structure has already yielded, higher modes still contribute to the internal forces of the structure. This means that when the contribution of higher modes of vibration is taken into account, the base shear of the structure keeps increasing even after yielding of the structure. In particular, for the considered bare frames, the contribution of the second and third modes of vibration is actually significant, while higher modes of vibration become negligible. If infill panels are included in the frame (Figures (c), (d), (g), (h)), the first mode of vibration is predominant regardless of the number of storeys, and the contribution of higher modes of vibration becomes negligible. Based on the previous considerations, it is sufficient to include three modes of vibration when the D-DAP is applied to RC framed structures with or without infill panels

\subsection{Calibration of the displacement step size}

Another crucial choice is the value of the increment of displacement within the loading step $\Delta_{\text {step }}$. In order to select the most appropriate step size, the frames have been analysed by the D-DAP with gradually reduced displacement step sizes. Three values of displacement step size have been considered: 10,1 and $0.1 \mathrm{~mm}$. Figure 4 shows the results of the analysis conducted for frames GL-1 and SR-1, with 3 and 9 storeys, with and without infill panels. When the step size is $10 \mathrm{~mm}$, sometimes the analysis stops before the target displacement $(5 \%$ of the total height of the frame) is reached, or fails at the very beginning. A value of $\Delta_{\text {step }}=1 \mathrm{~mm}$

$$
-\Delta_{\text {step }}=10.0 \mathrm{~mm} \quad---\Delta_{\text {step }}=1.0 \mathrm{~mm} \quad \Delta_{\text {step }}=0.1 \mathrm{~mm}
$$

Bare frame Infilled frame

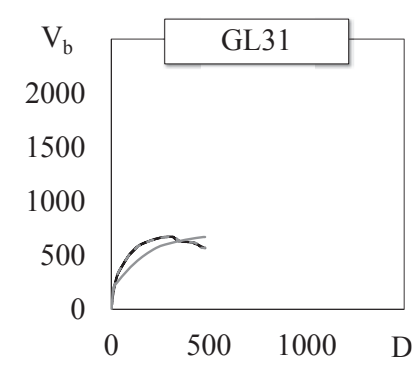

(a)

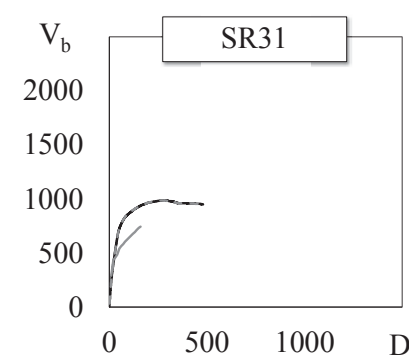

(e)

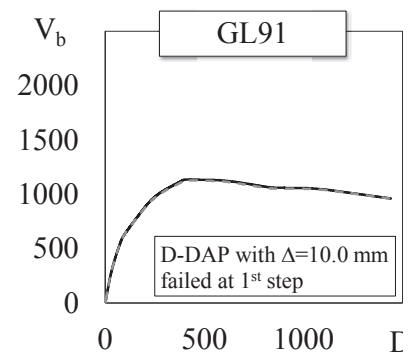

(b)

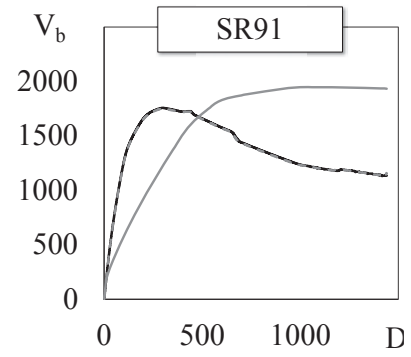

(f)

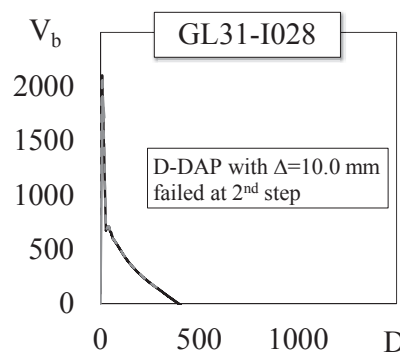

(c)

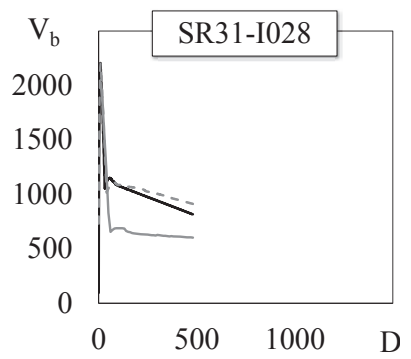

(g)

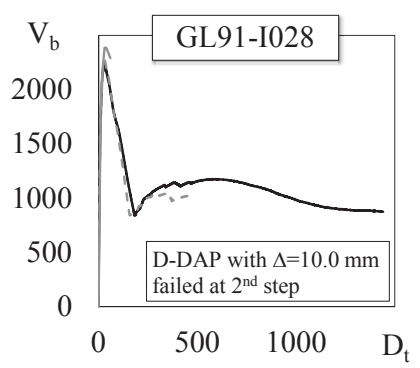

(d)

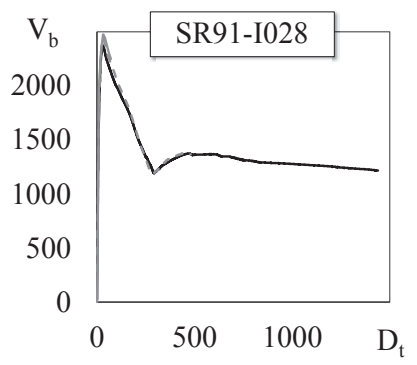

(h)

Figure 4 -Analysis of incremental step size for frame (a) GL31, (b) GL91, (c) GL31-I028, (d) GL91-I028 (e) SR31, (f) SR91, (g) SR31-I028, (h) SR91-I028 
avoids the untimely failure of the analysis, especially in the case of infilled frames (Figure (c), (d), (g), (h)) whereby the analysis with larger $\Delta_{\text {step }}$ stopped right after the attainment of the maximum strength of infills. Furthermore, the prediction of the seismic response provided by the D-DAP with $\Delta_{\text {step }}=1 \mathrm{~mm}$ is significantly different from that obtained with $\Delta_{\text {step }}=10 \mathrm{~mm}$, as showed in Figure 5 (a), (d), (e), (f), (g). No significant changes in the prediction of the seismic response were observed when the step size has been further reduced to $0.1 \mathrm{~mm}$. Since the tendency noted in the shown frames has been essentially observed in all other analysed frames, a value of $\Delta_{\text {step }}=1 \mathrm{~mm}$ could be considered appropriate. However, since the D-DAP with $\Delta_{\text {step }}=1 \mathrm{~mm}$ in very few cases failed slightly before the target displacement was reached, for the sake of accuracy the displacement step size has been assumed equal to $0.1 \mathrm{~mm}$.

\subsection{Calibration of the equivalent damping law}

The total equivalent viscous damping ratio is the summation of the inherent viscous damping in the elastic range $\xi_{0}$, and the viscous damping $\xi_{\text {hyst }}$ due to the hysteretic behavior [17, 18]. The damping ratio due to the hysteretic behaviour $\xi_{\text {hyst }}$ (hysteretic damping) is generally assumed as a function of the ductility demand $\mu$, according to the following form:

$$
\xi_{\text {hyst }}=\xi_{\infty}\left(1-\frac{1}{\mu^{\alpha_{1}}}\right)
$$

whereby $\xi_{\infty}$ represents the asymptotic value that the hysteretic damping reaches for infinite values of ductility demand, and $\alpha_{1}$ is a constant usually assumed equal to 0.5 or 1 . Here $\alpha_{1}$ is taken equal to 1 .

The goal of the numerical calibration is the determination of the asymptotic damping $\xi_{\infty}$ that is appropriate for the structural types under investigation. To this end, the average drift $\Delta_{m}$, defined as the summation of drifts at all storeys divided by the total number of storeys, is assumed as the reference parameter of the numerical calibration. Incremental Dynamic Analyses (IDA) are conducted for every case study frame, for PGAs increasing from $0.02 \mathrm{~g}$ to $2 \mathrm{~g}$, in steps of $0.02 \mathrm{~g}$. A set of ten artificial ground motions (EC8-Part 1 [19]), compatible with the EC8 elastic spectrum for soil type $\mathrm{C}$ and characterized by $5 \%$ damping ratio and reference PGA for soil type A equal to $0.35 \mathrm{~g}$, is adopted as reference seismic input. The accelerograms were generated by SIMQKE computer program [20] so that the mean spectrum in terms of acceleration is compatible with the response spectrum proposed by EC8 in Appendix A. For every level of seismic excitation and for each of the 10 ground motions, the maximum value during the time history of the average drift $\Delta_{m}$ is calculated. Then, the mean value of the average drift $\Delta_{m}$ over the 10 ground motions is calculated for each PGA. The value of $\Delta_{m}$ is calculated also at each step of the D-DAP analysis. Thus, every average drift $\Delta_{m}$ corresponds to a value of peak ground acceleration PGA determined by the D-DAP with an elastic response spectrum with fixed $5 \%$ damping. Generally, for a fixed $\Delta_{m}$, the D-DAP with $5 \%$ damping underestimates the values of PGA provided by the IDA. Hence, the PGA evaluated by the DDAP has to be corrected with an appropriate damping ratio. Thus, for every relevant frame, the optimal hysteretic damping $\xi_{\infty}$ is calculated as the value that minimizes the summation of the squares of the differences between the PGAs by D-DAP and that by IDA. For every case study frame, the optimization process led to a different value of optimal $\xi_{\infty}$.

In the second part of the calibration, the optimal values of $\xi_{\infty}$ obtained for every frame were plotted as a function of different parameters. Out of the considered parameters, the fundamental period $T_{1}$ showed the most significant influence on the value of $\xi_{\infty}$. In particular, in the case of very stiff structures the values of $\xi_{\infty}$ tend to increase with $T_{1}$ (Figure 5a). This ten- 


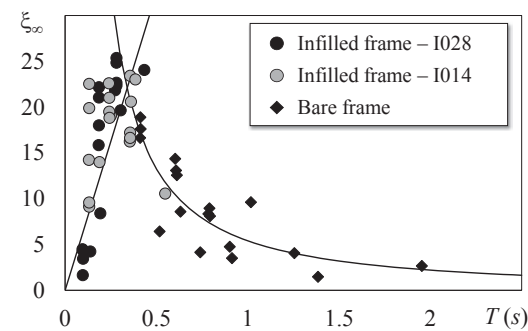

(a)

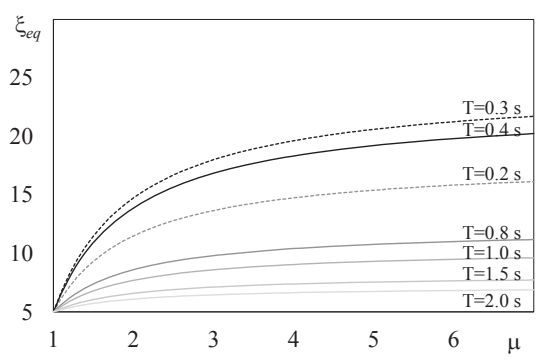

(b)

Figure 5 - (a) Relation between the optimized $\xi_{\infty}$ and the fundamental period $T$ for every case study frame; (b) Equivalent damping evaluated by the proposed law plotted for fixed value of $T_{1}$

dency is demonstrated by almost all the infilled case study frames, whose fundamental periods is smaller than $0.4 \mathrm{~s}$ (grey and black dots). This tendency is inverted for larger $T_{1}$, and the values of $\xi_{\infty}$ tend to decrease. This trend is exhibited by all the analysed bare frames (black diamonds). In fact, the lack of infill panels strongly reduces the stiffness of bare structures, which have fundamental periods ranging from 0.40 to $1.95 \mathrm{~s}$. Only two infilled frames fall in this region, and they are frame GL91-I028 and GL91-I014 with $T_{1}$ equal to 0.433 and $0.548 \mathrm{~s}$, respectively. Based on these results, the asymptotic damping ratio $\xi_{\infty}$ can be reasonably expressed as a function of the fundamental period of the structure.

To propose an equation for the evaluation of the asymptotic damping $\xi_{\infty}$, the domain $\xi_{\infty}$ $T_{1}$ has been divided into two regions. For low fundamental periods, the relation between $\xi_{\infty}$ and $T_{1}$ could be approximated by a linear function, whilst for larger fundamental periods it could be approximated by a hyperbolic function. The linear equation has been calibrated by minimizing the sum of the square deviations of values of $\xi_{\infty}$ determined by numerical analyses and by the proposed equation for frames whose fundamental period was lower than $0.40 \mathrm{~s}$. Instead, the hyperbolic function has been calibrated by minimizing the sum of the square deviations of values of $\xi_{\infty}$ in the case of frames with fundamental period higher than $0.40 \mathrm{~s}$. Hence, given a period $T_{1}$, the value of $\xi_{\infty}$ to be assumed is the minimum between the values provided by the linear and the hyperbolic equation, as expressed in the following function:

$$
\xi_{\infty}=\min \left(65 T_{1} ; \frac{5.4}{T_{1}^{1.3}}\right)
$$

The intersection between the two functions occurs for a period of vibration equal to $0.34 \mathrm{~s}$. In Figure 5(a) Equation 8 is plotted with the continuous black line. It can be seen that the calibrated function approximates with a good accuracy the tendency of the values of $\xi_{\infty}$. Finally, the equation of the proposed equivalent viscous damping ratio is:

$$
\xi_{e q}=\xi_{0}+\min \left(65 T_{1} ; \frac{5.4}{T_{1}^{1.3}}\right)\left(1-\frac{1}{\mu}\right)
$$

Figure 5 (b) plots the proposed equation of $\xi_{e q}$ as function of the ductility demand $\mu$. Each curve displays the results obtained for a different value of $T_{1}$. Regardless of $T_{1}$, the equivalent viscous damping ratio increases with the ductility demand $\mu$. However, when the ductility demand becomes larger, the rate of increase of $\xi_{e q}$ becomes smaller, and the equivalent viscous damping tends towards the asymptotic value $\xi_{0}+\xi_{\infty}\left(T_{1}\right)$.

\section{ANALYSIS OF THE RESULTS}

To validate the calibrated D-DAP, IDA was run to assess the seismic response of each case 
study frame. To this end, the numerical model described in Section 4.1 was subjected to the set of 10 ground motions introduced in Section 4.4. The PGA in IDA was increased in step of $0.02 \mathrm{~g}$ until the $5 \%$ of storey drift is attained at one of the storeys. For every PGA and for each ground motions, the maximum values during the time history of the following Engineering Demand Parameters (EDP) are evaluated: maximum top displacement $D_{t}$, maximum base shear $V_{b}$, maximum drift at each $i$-th storey $\Delta_{i}$ and the maximum average drift $\Delta_{m}$. The mean over the values of the 10 ground motions is calculated for each EDP and are assumed as the benchmark to evaluate the accuracy of the calibrated D-DAP.

For the evaluation of the seismic response at local level, three limit states were considered in the IDA, i.e. the attainment of a maximum storey drift equal to $1 \%, 2 \%$ and $4 \%$, which correspond to the attainment of the Immediate Occupancy (IO) limit state, the Life Safety (LS)
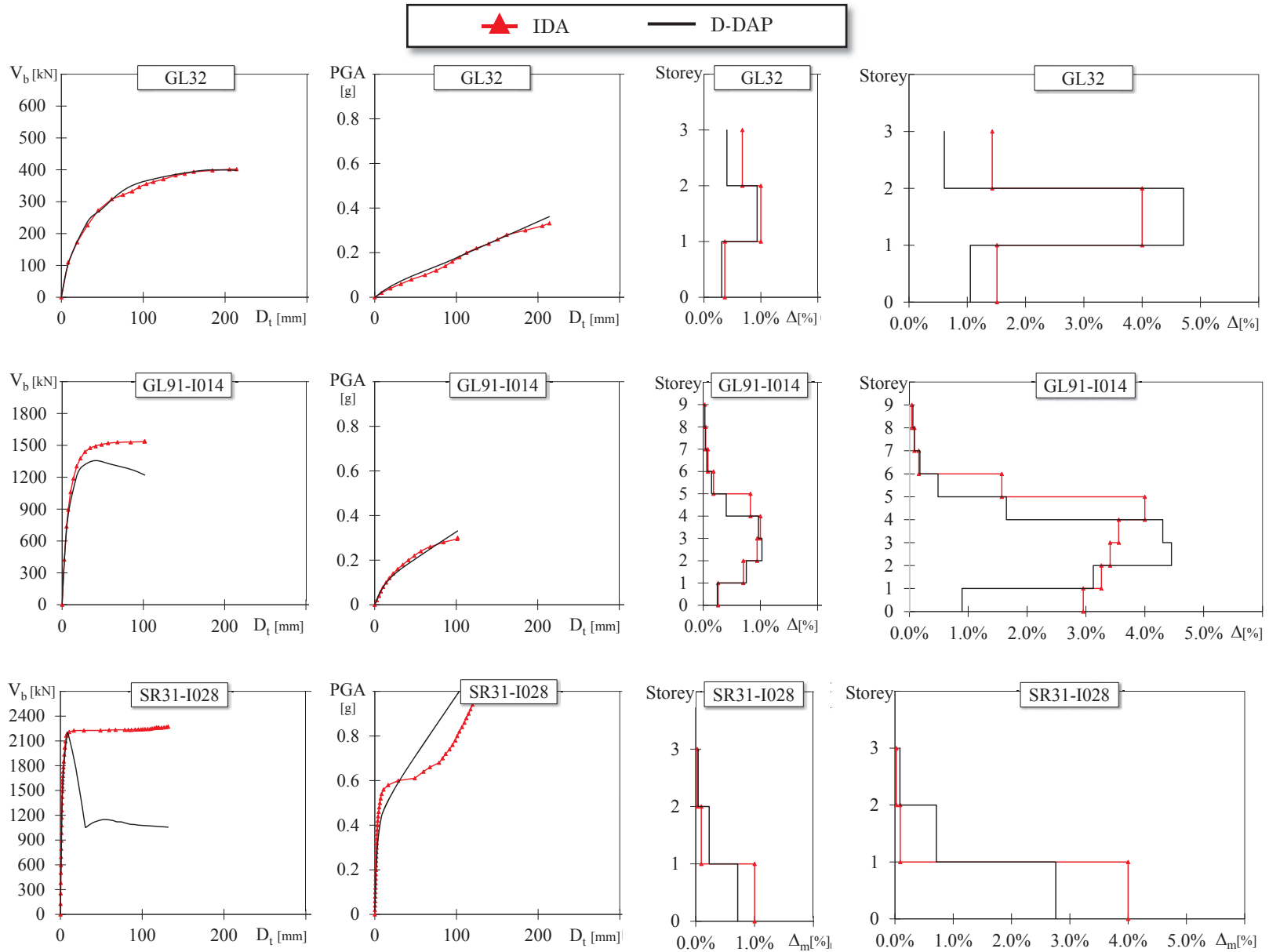

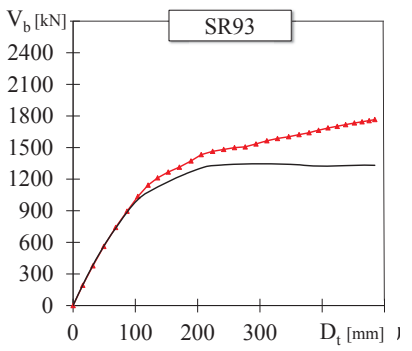

(a)

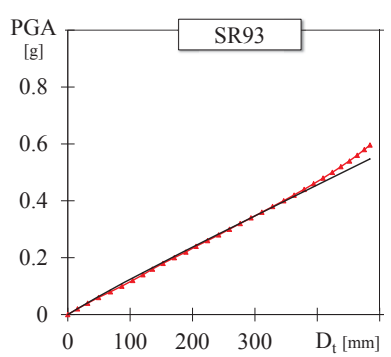

(b)

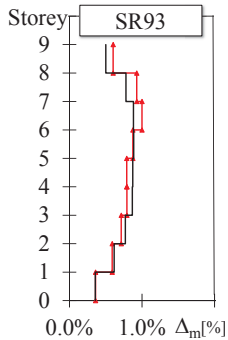

(c)

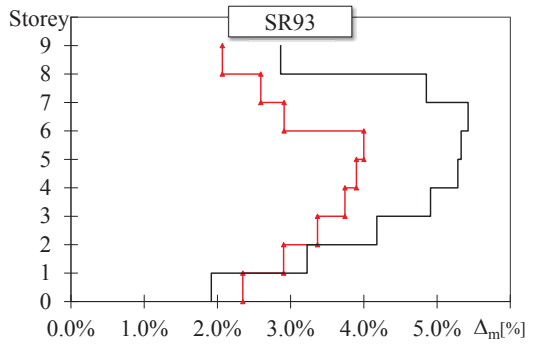

(d)

Figure 6 - Seismic performance of frames GL32, GL91-I014, SR31-I028 and SR93 in terms of (a) $V_{b}-D_{t}$, (b) PGA$D_{t}$, (c) distribution of drifts corresponding to the PGA leading to $1 \%$ maximum drift in the ID, (d) distribution of drifts corresponding to the PGA leading to $4 \%$ maximum drift in the IDA. 
limit state and the Near Collapse (NC) limit state, respectively. Given the PGA corresponding to the attainment of the considered limit state in the IDA, the corresponding distribution of storey drift has been evaluated by the D-DAP. For the sake of simplicity, the results are here presented for four significant frames. To cover the widest possible range of behaviour, the four selected frames included frames with 3 and 9 storeys, with large, medium and low gravity loads resting on beams, with and without infills. The frames thus picked are: GL32, GL91014, SR31-028 and SR91.

The first column of Figure 6 shows the seismic response of the considered frames in terms of $V_{b}-D_{t}$. It can be observed that the D-DAP provides an accurate estimate of the seismic behaviour of the considered bare frames (plots in first and last row of Figure 6). In those cases, the maximum base shear increases with the top displacement, and the D-DAP follows the trend of the IDA, with an error lower than the $18 \%$. In the case of infilled frames, an almost constant branch is observed in the $V_{b}-D_{t}$ curve determined by the IDA. This upper value of the base shear is due to the fact that for every PGA the maximum base shear is recorded in the IDA. Thus, once the infills have cracked, the displacement keeps increasing while the maximum base shear maintains almost the same value. This constant branch represents an upper limit for the base shear and is here interpreted as the ultimate lateral strength of the frame. The D-DAP estimates accurately the elastic behaviour predicted by the IDA and provides an abrupt reduction of the base shear force right after the cracking of the infills. Indeed, for such degrading structures, the D-DAP evaluates the maximum resistance with an error lower than $15 \%$. In the second columns of Figure 6, the PGAs associated to the displacement demands by the D-DAP are compared to the values predicted by the IDA. It can be observed that the D-DAP leads to an accurate prediction of the displacement demand regardless of the presence of infill panels.

Eventually, Figure 6 shows the distribution of storey drifts along the height for two of the considered limit cases, i.e. 1\% storey drift (column c) and 4\% storey drift (column d), respectively. At 1\% limit state, the D-DAP estimates the maximum storey drift with an error lower than 5\% for GL91-I014 and 35\% for SR31-I028. For medium and large storey drifts (i.e. 2\% and $4 \%$ limit state), the prediction of storey drifts of the IDA obtained by the proposed DDAP still has an acceptable level of accuracy for the bare frames. Particularly, in the case of infilled frames, the error committed by the D-DAP in evaluating the maximum drift is lower than $12 \%$ and $31 \%$ for frame GL91-I014 and SR31-I028, respectively.

Even though it is not shown in figure, given the PGA corresponding to the $4 \%$ limit state in the IDA, the D-DAP predicted the average drift $\Delta_{m}$ with less than $10 \%$ of error for frame GL32. For frame SR93 the error reached a value around 35\%, In the case of infilled frames, the error committed by the D-DAP is lower than 14\% and 20\% for frame SR31-I028 and GL91-I014, respectively. The tendency showed by those cases study can be found generally in all the other case study frames.

\section{CONCLUSIONS}

The research developed in three steps. First, the parameters ruling the operations of the D-DAP, i.e. The number of modes to be enveloped in the loading vector and the size of the displacement increment, were calibrated. To this end, two analyses were conducted and for the considered frames it was found that (i) the contribution of the modes of vibration higher than the third one is negligible and (ii) a displacement increment of $0.1 \mathrm{~mm}$ has to be assigned at each step to get accurate results for all the considered frames at reasonable computational cost. Afterwards, a new damping law was formulated by an optimization process that minimizes the differences between the results provided by the D-DAP and those obtained by the IDA.

The new equivalent damping law is a function of both the ductility demand and the fundamental period of vibration. According to the proposed equation, for very stiff structures, the value of $\xi_{\text {eq }}$ 
is linearly dependent from $T_{1}$. Instead, for more flexible structures, the relation between $\xi_{e q}$ and $T_{1}$ is hyperbolic. The effectiveness of the proposed D-DAP with the proposed damping law is investigated and the following conclusions can be drawn:

- As for the seismic response in terms of $V_{b}-D_{t}$, the D-DAP with the proposed damping law predicted the maximum base shear with an error on average equal to $20 \%$ for bare frames, and $15 \%$ for infilled frames. The seismic response in terms of $a_{g}-D_{t}$ was predicted accurately by proposed D-DAP, as well.

- The proposed D-DAP predicted the average drift at $4 \%$ limit state of infilled frames with an error lower than $20 \%$.

\section{REFERENCES}

[1] S.A. Freeman, J.P. Nicoletti, J.V. Tyrell, Evaluations of existing buildings for seismic risk - A case study of Puget Sound Naval Shipyard, Bremerton, Washington, in proceedings of 1st U.S. National Conf. on Earthquake Engineering, Earthquake Engineering Research Institute, Berkeley, 13-122, 1975.

[2] P. Fajfar, Capacity spectrum method based on inelastic demand spectra, Earthquake Engineering and Structural Dynamics, 28: 979-993, 1999.

[3] S. Antoniou, R. Pinho, Development and verification of a displacement - based adaptive pushover procedures, Journal of Earthquake Engineering, 8: 643-661, 2004.

[4] R. Monteiro R, C. Casarotti, Using the adaptive capacity spectrum method for seismic assessment of irregular frames, In proceedings of the 5th European workshop on the seismic behaviour of irregular and complex structures, Catania, Italy, 2008.

[5] A. Ghersi, P. Lenza, M. Pellecchia, Uno sviluppo Multimodale ed adattivo del metodo N1 per la verifica ed il progetto delle strutture intelaiate in c.a. 15th ANIDIS Conference, Padova, Italy. (in Italian) 2013

[6] P. Lenza, A. Ghersi, E. M. Marino, M. Pellecchia, A multimodal adaptive evolution of the N1 method for assessment and design of RC framed structures, Earthquake and Structures, 12, 2017.

[7] F. Barbagallo, M. Bosco, A. Ghersi, E. M. Marino, An overdamped multimodal adaptive nonlinear static procedure for seismic assessment of infilled r.c. buildings. Engineering Structures, 229, 2021.

[8] Italian Ministry of Public Works: Ministry Decree, 30/05/1974, Technical regulations for constructions with reinforced concrete, prestressed concrete and steel structure, Gazzetta Ufficiale Serie generale, 29/07/1974, Rome. (in Italian)

[9] Royal Decree n. 2229, 16/11/1939, Regulations for constructions of concrete and reinforced concrete. Supplemento Gazzetta Ufficiale n. 92, 18/04/1940, Rome. (in Italian)

[10] Italian Ministry of Public Works: Ministry Decree, 16/01/1996, Regulations for constructions in seismic areas, Gazzetta Ufficiale Serie generale, 5/02/1996, Rome. (in Italian)

[11] S. Mazzoni, F. McKenna, M. H. Scott, G. L. Fenves, B. Jeremic, OpenSees command Language Manual, Pacific Earthquake Engineering Research Center, University of California at Berkely, USA, 2003.

[12] J.B. Mander, M.J.N. Priestley, R. Park, Theoretical stress strain model for confined concrete Journal of Structural Engineering ASCE, 114:1804-1825, 1988

[13] F. Barbagallo, M. Bosco, E. M. Marino, P.P. Rossi, On the fibre modelling of beams in RC framed buildings with rigid diaphragm, Bulletin of earthquake engineering, 18:189-210, 2020

[14] T. B. Panagiotakos, M.N. Fardis, Seismic response of infilled RC frame structures, in proceedings of the 11th world conference on earthquake engineering, 1996.

[15] D. Celarec, P. Ricci, M. Dolšek, The sensitivity of seismic response parameters to the uncertain modelling variables of masonry-infilled reinforced concrete frames, Engineering Structures, 35, 165-177, 2012 
[16] M. Calvi, S. Santini, Preliminary tests in infill masonry, PREC8 Progress Report, University of Pavia, Department of structural mechanics, Pavia, 1994

[17] C.A. Blandon, M.J.N. Priestley, Equivalent viscous damping equations for direct displacement based design, Journal of earthquake engineering, 9: 257-278, 2005

[18] M.J.N. Priestley, 2003, Myths and fallacies in earthquake engineering, The Mallet Milne Lecture, IUSS Press, Pavia, Italy.

[19] CEN. Eurocode 8: Design of structures for earthquake resistance - Part 1: Design of seismic resisting buildings, EN 1998-1. European Committee for Standardization: Bruxelles, Belgium, 2005.

[20] SIMQKE. A program for artificial motion generation, User's manual and documentation, Department of Civil Engineering MIT, 1976. 